\title{
Primary thrombocythaemia following splenectomy
}

\author{
J. R. O'DONNELL* \\ M.B., Ch.B., M.R.C.P.I. \\ P. A. M. BAILEY $\dagger$ \\ B.A., M.B., Ch.B., M.R.C.Path.
}

\author{
J. G. ERSKINE* \\ M.B., Ch.B., M.R.C.P.(UK), M.R.C.Path. \\ J. F. DAVIDSON* \\ M.B., Ch.B., F.R.C.P.(Edin.), M.R.C.Path.
}

Departments of Haematology, *Glasgow Royal Infirmary, and + Dumfries and Galloway Royal Infirmary

\section{Summary}

A 20-year-old male who required splenectomy following abdominal trauma was subsequently shown to have primary thrombocythaemia. This report illustrates the diagnostic problems associated with a refractory post-splenectomy thrombocytosis, and the therapeutic difficulties in the management of primary thrombocythaemia are discussed.

\section{Introduction}

Primary thrombocythaemia (PT) is a well recognized syndrome within the spectrum of myeloproliferative disorders. Typically presenting from middle-age onwards, difficulties may arise in differentiation from secondary thrombocytosis or other myeloproliferative states particularly in younger patients. This report of an unusual presentation of PT in a 20-year-old male demonstrates some of the problems associated with the diagnosis and management of the disorder.

\section{Case report}

A 20-year-old healthy male who had worked for 4 years in a nuclear reactor plant was involved in a road traffic accident and required a laparotomy because of intra-abdominal trauma. At operation a large haemoperitoneum consequent upon a ruptured spleen was found and splenectomy was performed. The spleen was noted to be enlarged, weighing $425 \mathrm{~g}$, and operative blood loss was copious, being estimated at $>3$ litres. Immediate postoperative progress was uncomplicated. A pre-operative blood count was not available and so a platelet count of $1016 \times 10^{9} / 1$, immediately following splenectomy appeared unremarkable. Over the next 10 days the platelet count rose progressively to $3472 \times 10^{9} / 1$. The thrombocytosis was assumed to be an unusually marked reactive response following trauma and splenectomy and was expected to subside rapidly. Oral salicylate therapy was, however, started as prophylaxis against possible thrombotic compliồ cations.

Over the next 6 weeks the platelet count remaine markedly elevated between $2976 \times 10^{\circ} / 1$ and $3312 \times 9$ $10^{9} / 1$, although the patient remained asymptomatic. Because of persistent marked thrombocytosis furthet investigations were initiated. Serial platelet counts over 7 days ranged from $2950 \times 10^{\circ} / 1$ to $4410 \times 10^{\circ}$ 要 (mean value $3760 \times 10^{\circ} / 1$ ). Haemoglobin levels were normal. There was a leucocytosis of $18.9 \times 10^{9} /$ tot $^{\circ}$ $21.9 \times 10^{9} / 1$ (approximately $70 \%$ polymorphs). Blof $\overrightarrow{\mathrm{d}}$ films showed post-splenectomy changes with marke variation in platelet morphology and occasionas megakaryocyte fragments. Sternal bone marrov aspirate revealed an extremely hypercellular marrowo Granulopoiesis was hyperplastic and there was \& gross increase in megakaryocytes which forme distinct clumps. Many giant megakaryocytes were noted showing nuclear hyperploidy. A bone biops was followed by profuse bleeding for several hours. from the posterior iliac crest site. Histology showe a similar picture to the marrow aspirate with large. clumps of megakaryocytes. There was no significan? increase in reticulin. Chromosome analysis showed normal male karyotype. Leucocyte alkaline phospha? tase score was normal as were blood volume studies and serum vitamin $B_{12}$ assay. Platelet function tests revealed only salicylate inhibition of aggregation Biochemical investigations showed hyperkalaemia of $6.4 \mathrm{mmol} / 1$, hyperuricaemia of $450 \mu \mathrm{mol} / 1$ and 8 slightly elevated lactic dehydrogenase of $886 \mathrm{u} / \mathrm{f}_{\mathrm{f}}$ Blood volume studies were not performed.

The diagnosis of primary thrombocythaemia wa well established despite the unusual age and presen? tation of the patient. Radioactive phosphoruse $3 \mathrm{mCi}$, was administered and salicylate therapy was continued. The platelet count had fallen to $1808 x^{+}$ $10^{9} / 1$ after 2 months but rose over the following month to $2544 \times 10^{9} / 1$. A further $2 \mathrm{mCi}$ of radioactive phosphorus were administered and within 6 weeks 
the platelet count had fallen $\left(1228 \times 10^{9} / 1\right)$. However, one month later the count had risen to $2032 \times$ $10^{9} / 1$.

\section{Discussion}

The distinction between primary thrombocythaemia and a reactive thrombocytosis may often prove difficult. However, a platelet count in excess of $2000 \times 10^{9} / 1$ for $>2$ months after splenectomy is highly suggestive of primary thrombocythaemia (Williams, 1977). A lesser degree of thrombocytosis (up to $1000 \times 10^{9} / 1$ ) may persist for longer periods in patients where anaemia continues following splenectomy (Hirsh and Dacie, 1966). Post-splenectomy thrombocytosis could therefore be dismissed as simply reactive, although it has been reported that splenectomy can uncover unrecognized primary thrombocythaemia (Hardisty and Wolff, 1955; Bensinger, Logue and Rundles, 1970).

This case clearly showed the diagnostic problem with primary thrombocythaemia, especially in very young patients. However, the degree and duration of the thrombocytosis combined with the very characteristic morphological appearances and signs of increased cell turnover are virtually diagnostic of primary thrombocythaemia. Although accurate platelet counting is difficult at such concentrations, the inhibitory effect of salicylates on platelet aggregation may have aided counting reproducibility. Unfortunately, this effect also prevented demonstration of the abnormalities of platelet function known to occur in primary thrombocythaemia (Hardisty and Wolff, 1955; McClure et al., 1966).

Information regarding the natural history of primary thrombocythaemia is scanty, but significant morbidity and mortality from thrombosis and/or haemorrhage has been recorded, especially following splenectomy (Gunz, 1960; Silverstein, 1968). Because of that risk, salicylate therapy was instituted in this patient. Urgent myelosuppressive therapy has been advised in patients where primary thrombocythaemia becomes apparent after splenectomy, and agents such as radioactive phosphorus and cytotoxic drugs are effective for control of primary thrombocythaemia, particularly in the elderly (Bensinger et al., 1970). However, in the very young patient there is little indication from the few cases reported on the long-term efficacy or risks of myelosuppressive therapy. The response of the present patient to therapy with radioactive phosphorus has not been encouraging thus far.

Five cases of primary thrombocythaemia have been reported in patients under 20 years of age (Thieffry, Buhot and Aicardi, 1975; Levinson et al., 1958; Ozer et al., 1960; Spach, Howell and Harris, 1963; Fickers and Speck, 1974). The course in these young patients was very variable. One patient who presented at 8 years of age remained healthy without treatment 7 years after initial diagnosis of primary thrombocythaemia (Spach et al., 1963). However, 2 other patients developed acute myeloid leukaemia within 3 years of diagnosis, one after treatment with radioactive phosphorus, the other after chemotherapy with thiotepa and busulphan. Estimates vary widely on the frequency of blastic transformation in myeloproliferative states (Ward and Block, 1971). The scarcity of reports on primary thrombocythaemia, especially in the young, makes it impossible to quantify this risk. An added problem is the requirement for myelosuppressive therapy, in itself almost certainly leukaemogenic.

This unusual case of primary thrombocythaemia with gross elevation of the platelet count following splenectomy in a very young patient illustrates some of the diagnostic and therapeutic difficulties in this disorder. As thrombocytosis is an expected postsplenectomy finding, close haematological monitoring of a prolonged thrombocytosis is essential to identify the rare patient with an underlying myeloproliferative disorder.

\section{References}

Bensinger, T.A., Logue, C.L. \& Rundles, R.W. (1970) Hemorrhagic thrombocythemia: control of postsplenectomy thrombocytosis and Melphalan. Blood, 36, 61 .

FICKERS, M. \& SPECK, B. (1974) Thrombocythaemia: Familial occurrence and transition into blastic crisis. Acta haematologica, 51, 257.

GuNZ, F.W. (1960) Hemorrhagic thrombocythemia: a critical review. Blood, $15,706$.

HARDISTY, R.M. \& WolfF, H.H. (1955) Haemorrhagic thrombocythaemia: a clinical and laboratory study. British Journal of Haematology, 1, 390.

Hirsh, J. \& DACIE, J.V. (1966) Persistent post-splenectomy thrombocytosis and thromboembolism: a consequence of continuing anaemia. British Journal of Haematology, 12, 44.

Levinson, B., Jones, R.S., Wintrobe, M.M. \& Cartwright, G.E. (1958) Thrombocythemia and pulmonary intraalveolar coagulum in a young woman. Blood, 13, 959.

MCCluRe, P.D., IngRaM, G.I.G., STACEY, R.S., Glass, U.H. \& MATCheTT, M.O. (1966) Platelet function tests in thrombocythaemia and thrombocytosis. British Journal of Haematology, 12, 478.

Ozer, F.L., TruaX, W.E., Miesch, D.C. \& Levin, W.C. (1960) Primary hemorrhagic thrombocythemia. American Journal of Medicine, 28, 807.

Silverstein, M.N. (1968) Primary or hemorrhagic thrombocythemia. Archives of Internal Medicine, 122, 18.

SpaCh, M.S., Howell, D.A. \& Harris, J.S. (1963) Myocardial infarction and multiple thromboses in a child with primary thrombocytosis. Pediatrics, 31, 268.

Thieffry, S., Buhot, S. \& AICARdi, J. (1957) Une observation de thrombocythémie essentielle. Sang, 28, 264.

WARD, H.P. \& BLOCK, M.H. (1971) The natural history of agnogenic myeloid metaplasia (AMM) and a critical evaluation of its relationship with the myeloproliferative syndrome. Medicine, 50, 357.

Williams, W.J. (1977) Thrombocytosis. In: Haematology (Ed by Williams, W.J., Beutler, E., Erslev, A.J. \& Rundles, R.W.), 2nd edn, p. 1364. McGraw Hill Book Company, New York. 